Table 1. Degeneration in the half-side section of the 2nd lumbar segment

\begin{tabular}{llccccc}
\hline \multirow{2}{*}{ Nerves Observed } & \multicolumn{5}{c}{ Degeneration } \\
\cline { 2 - 6 } & $\begin{array}{c}\text { Small- } \\
\text { sized }\end{array}$ & $\begin{array}{c}\text { Medium- } \\
\text { sized }\end{array}$ & $\begin{array}{c}\text { Large- } \\
\text { sized }\end{array}$ & $\begin{array}{c}\text { Maximum- } \\
\text { sized }\end{array}$ & $\begin{array}{c}\text { Total } \\
\text { No. }\end{array}$ \\
\hline Spinal & Dorsal roots & 0 & 0 & 0 & 0 & 0 \\
Nerves $\quad$ Ventral roots & 104 & 24 & 7 & 0 & 135 \\
N. splanchnicus inferior & 101 & 22 & 0 & 0 & 123 \\
N. hypogastricus & 14 & 3 & 0 & 0 & 17 \\
\hline
\end{tabular}

degeneration of fibers in the $S_{1}-S_{3}$ ventral roots, and little in the $S_{1}-S_{3}$ dorsal roots was observed. Furthermore, degenerated fibers in the pelvic nerve, and the vesical nerve were found significantly. These were small- and medium-sized fibers, respectively (Table 2). The results following the one-side section of the

Table 2. Degeneration in the half-side section of the 1st sacral segment

\begin{tabular}{lcrrrrr}
\hline \multirow{2}{*}{ Nerves Observed } & \multicolumn{5}{c}{ Degeneration } \\
\cline { 2 - 7 } & $\begin{array}{c}\text { Small- } \\
\text { sized }\end{array}$ & $\begin{array}{c}\text { Medium- } \\
\text { sized }\end{array}$ & $\begin{array}{c}\text { Large- } \\
\text { sized }\end{array}$ & $\begin{array}{c}\text { Maximum- } \\
\text { sized }\end{array}$ & $\begin{array}{c}\text { Total } \\
\text { No. }\end{array}$ \\
\hline Spinal & Dorsal roots & 6 & 1 & 0 & 0 & 7 \\
$\quad$ Nerves & Ventral roots & 248 & 116 & 60 & 10 & 434 \\
N. pelvicus & 239 & 107 & 0 & 0 & 346 \\
Vesical Nerves & 146 & 7 & 0 & 0 & 153 \\
\hline
\end{tabular}

anterior horn of the spinal cord were almostly similar to those of the proceedings.

With these experiments, it was clarified that many degenerated fibers in the peripheral nerves were recognized following spinal injuries, and these fibers mainly originated in ventral roots. It was also noted that degeneration of small-sized fibers in ventral roots so as to the dorsal roots was to be related with the symptoms of autonomic nerves recognized after the spinal injuries.

\title{
14. Pathophysiological Studies on the Anastomosis of the Nerve The 1st Report
}

Hiroaki Kawakita, Kazuo Nishimura, Norikazu Sawa, Kiyoshi Uematsu, Syoji Bando, Tuyoshi Fujimoto, Ken Ito and Hiro Toyohara The Fujita's Surgical Clinic, Kobe Medical College

Studies on the anastomosis of the nerves were made by methods using the action potential tracing of nerves and the phase microscopic observation in dogs.

The results obtained were as follows:

1. In regard to the difference of regeneratic ability between the thickness of the nerve fibers and the kind of neurons. 
2. Relation to the degree of injury in the peripheral nerves and its regenerative ability.

3. Comparison with the use of the tantalum sheet and the auto-blood vessels.

Furthermore, the possibility of crossanastomosis of autonomic nerves was found out and in these cases, functional changes of the abdominal cavity and the effect on the conducting-pathway in the visceral sensation were estimated.

\title{
15. Electroencephalographic Observations and Clinical Effects of Craniotomy for Craniosynostosis
}

\author{
Kazuhisa Nagate, Shozaburo Utsumi, Yutaka Hattori, \\ Chikayoshi TERADA AND Yutaka HorI \\ The 2nd Surgical Department, Nara Medical College
}

\begin{abstract}
Although a great deal has been written about surgical treatment of craniosynostosis, man can find few works of electroencephalographic studies. Especially on the electroencephalographic report of the operation effects of this disease, there is much that remains obscure.

The authors observed electroencephalographically the process before and after craniotomy in 15 cases of craniosynostosis.

1) From craniosynostosis infants were mostly recorded infantile abnormal findings; spikes and slow waves, involving 3 cases of hypsarhythmia with nodding seizure. One case that did not show remarkable abnormality was diagnosed from clinical findings and operation effect as microcephalia.

2) These abnormal EEG patterns were markedly improved by craniotomy, namely spikes and slow wave factors, particularly the latter diminished and normal appearance took place. According to these electroencephalographic changes, clinical findings i.e. fixability of neck, abnormal muscle tonus in extremities, ocular symptoms etc. were also improved. Needless to say that clinical and electroencephalographic effects were remarkable, if craniotomy was accomplished within 2 life years.

3) Therefore, the authors mention that craniotomy should be applied at the most abnormal part of cranium in electroencephalographic findings or cranial shape.
\end{abstract}

\section{Hydroencephaldysplasia}

Tetsu Mitsumune, Kowichi Kitamura and Shiro Hayashi Department of Neurosurgery, Tokyo University School of Medicine

One of us reported a case of hydroencephalodysplasia at the 15th Annual 